\title{
Small-angle Scattering of $\mathrm{X}$-rays and Neutrons as Structural Tools in Nanoscience: Block Copolymer Micelles, Protein-detergent Complexes, Microgels and other Nanoparticles
}

\author{
Jan Skov Pedersen ${ }^{1 *}$ \\ ${ }^{1)}$ Department of Chemistry and Interdisciplinary Nanoscience Center (iNANO), Aarhus University, Gustav \\ Wieds Vej 14, 8000 Aarhus C, Denmark \\ ${ }^{*}$ E-mail jsp@chem.au.dk
}

Small-angle X-ray scattering (SAXS) and small-angle neutron scattering (SANS) are excellent experimental tools for obtaining structural information on macromolecules, selfassembled structures, and nanoparticles in solution. The techniques give information on shape, size and organization of particles and molecules of sizes up to hundreds of nanometers with sub-nm resolution. It also provides key parameters such as mass, maximum diameter and radius of gyration. In contrast to many other techniques, SAXS and SANS does not require any special sample preparation or deuteration, and can be performed for almost any solvent/buffer condition, however, $\mathrm{D}_{2} \mathrm{O}$ is usually used in the buffers for SANS as it enhances contrast and reduce background. Furthermore, the studied assemblies or particles may be amorphous or crystalline, may have disordered region or randomness, and may have (smaller) variation in size and shape without spoiling the possibility of structure determination. However, deriving detailed structural information from SAXS data requires sophisticated analysis approaches [1,2]. There has been a large progress in analysis within the last decades and this has led to broad field of applications of the SAXS and SANS methods. In this lecture, the basic principles and theory will be described and some recent applications to block copolymer micelles [3], protein-detergent complexes [4] and microgels [5] will be presented.
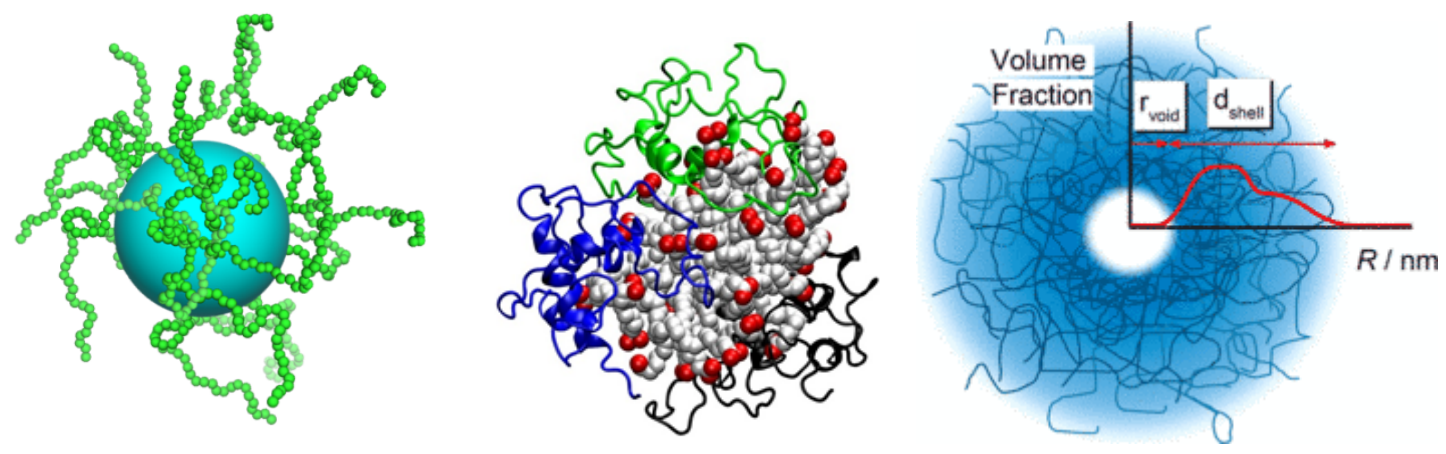

Figure 1. Examples of structures: Block copolymer micelles [3], protein-detergent complexes [4] and hollow microgel [5].

\section{References.}

[1] Pedersen J.S. (1997) Adv. Colloid Interface Sci. 70: 171-210.

[2] Pedersen J.S. in "Neutrons, X-rays and light: scattering methods applied to soft condensed matter": Eds.: P. Lindner and T. Zemb, 2002, North Holland. 
[3] Dai J., Alaei Z., Plazzotta B., Pedersen J.S., Furo I. J. Phys. Chem. B, 2017, 121 (45), pp 10353-10363

[4] Nedergaard Pedersen J., Frederix, P.W.J.M., Pedersen, J.S.; Marrink S.J.; Otzen D. ChemBioChem, 19 (3), 2018, pp 263-271.

[5] Dubbert J., Honold T., Pedersen J.S. Radulescu A., Drechsler M., Karg M., Richtering W. Macromolecules, 47 (24), 2014, pp 8700-8708. 